\title{
Multisensor Integration for Declaring the Odor Source of a Plume in Turbulent Fluid-Advected Environments
}

\author{
Wei Li \\ Department of Computer Science \\ California State University, Bakersfield \\ Bakersfield, CA 93311, U.S.A. \\ wli@cs.csubak.edu \\ Carl Bloomquist \\ Department of Computer Science \\ California State University, Bakersfield \\ Bakersfield, CA 93311, U.S.A.
}

\author{
Mohamoud M. Elgassier \\ Department of Computer Science \\ California State University, Bakersfield \\ Bakersfield, CA 93311, U.S.A. \\ mmellgassier@cs.csubak.edu \\ Karan Srivastava \\ Department of Computer Science \\ California State University, Bakersfield \\ Bakersfield, CA 93311, U.S.A.
}

\begin{abstract}
A mission of chemical plume tracing (CPT) is to navigate an autonomous underwater vehicle (AUV) to find a chemical plume, to trace the plume to its source, and to declare the source location. This paper presents a multisensor integration strategy for source declaration subsumed in a behavior based control architecture with several individual behaviors. The performance of the proposed strategy is evaluated using a simulated turbulent fluid environment. The results show that, for 1000 test runs, the proposed strategy reaches a success rate in declaring the odor source of $98 \%$ and an average error of the declared source location about 3.27 meters in an operation area with length scales of $\mathbf{1 0 0}$ meter. Source verification is developed using a fuzzy reasoning based segmentation algorithm to recognize the odor source in color images acquired by a visual sensor in the vicinity of the odor source.
\end{abstract}

Index Terms - Autonomous vehicles, behavior-based control, chemical plume tracing, multiple sensor integration, subsumption architecture.

\section{INTRODUCTION}

AN emerging research topic in intelligent systems is to design an autonomous system to search for environmentally interesting phenomena, unexploded ordnance, undersea wreckage, and sources of hazardous chemicals or pollutants in real world. Such a problem is referred as to Chemical Plume Tracing (CPT).

Practical strategies attempted to solve the CPT problem based on flow information and instantaneous chemical detections. Belanger and Willis [1] presented plume tracing strategies intended to mimic moth behavior and analyzed the performance in a computer simulation. Li et al. [2] developed, evaluated, and optimized passive and active counter-turning strategies inspired by moth behaviors, that allow an autonomous vehicle to trace a chemical plume with significant meander from a possible large initial distance to the odor source. Grasso et al. [3], [4] evaluated biomimetic strategies, challenged theoretical assumptions of the strategies by implementing biomimetic strategies on their robot lobster, and compared three variants of plume tracing strategy by using a single-sensor or two sensors detecting fluoresce. Farrell et al. [5] adopted hidden Markov methods to estimate a chemical plume by constructing a plume map in an operation area with length scales of $100 \mathrm{~m}$. Robots that attempt plume tracing in laboratory environments were described in [6]-[10]. The works in [11]-[13] discussed multi robot strategies for plume tracing and estimation. The passive and active plume tracing strategies proposed in [2] were implemented on an underwater vehicle, and their in-water test runs conducted in November 2002 on San Clemente Island, CA in [14], and in June 2003 at Duck, $\mathrm{NC}$ in [15], respectively.

In order to declare the odor source in turbulent fluidadvected environments, an AUV is assumed to be equipped with multiple sensors to detect chemical concentration, to sense fluid flow velocity and direction, and to measure its current location. This paper presents a multisensor integration strategy for source declaration. The strategy described herein is to construct a source identification zone based on last chemical detection points (LCDPs) in the order of time series during CPT missions, known as SIZ_T. The mean value of LCDPs is declared the odor source, if the SIZ_T size becomes small enough. The simulation studies show that, for 1000 test runs, the strategy reaches a success rate in declaring the odor source of $98 \%$ and an average error of the declared source location about $3.27 \mathrm{~m}$ in a simulated turbulent fluid environment with length scales of $100 \mathrm{~m}$. Source verification is developed using a fuzzy reasoning based segmentation algorithm to recognize the odor source in color images acquired by a visual sensor in the vicinity of the odor source.

\section{SUBSUMPTION ARCHITECTURE}

The multisensor integration strategy for source declaration presented in this paper is subsumed in a behavior based control architecture with several individual behaviors, as shown in Fig. 1. Each of the layers in the architecture can be 


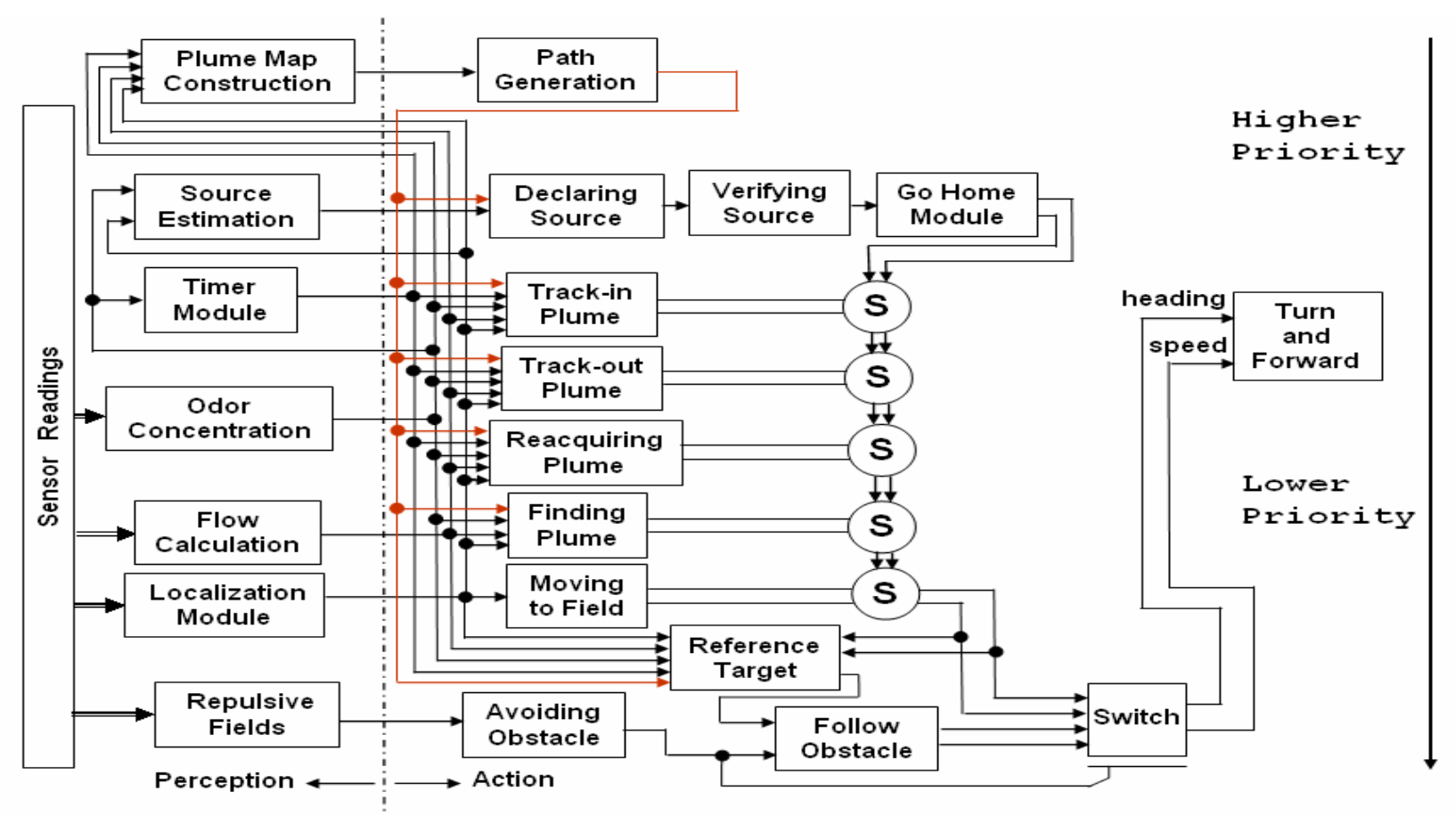

Fig. 1. Subsumption architecture for multisensor-based chemical plume tracing.

viewed as an abstract behavior for a particular task. A behavior can be viewed as a mapping of sensory inputs to a pattern of motor actions which then are used to achieve a task. The behavioral layers operate concurrently and independently. The subsumption architecture is composed of six fundamental behaviors: find a plume (Find-Plume), maintaining the plume (Maintain-Plume), reacquire the plume (Reacquire-Plume), declaring the source location (Declare-Source), avoiding an obstacle (Avoid-Obstacle), and constructing a plume map (Construct-Plume-Map). Maintain-Plume and ReacquirePlume are abstracted by the location of pheromone-emitting females by flying male moths [16], [17]. Find-Plume is abstracted from fluid mechanics forces in [18], [19]. Construct-Plume-Map and Avoid-Obstacle are discussed in [5] and [20], respectively. This paper will discuss the DeclareSource issue. For CPT, the control commands from the architecture to the vehicle guidance are speed and heading commands. In the architecture, the outputs from a higher layer can override, or subsume, the output from behaviors in the next lower layer using inhibition or suppression mechanisms to coordinate potential conflicts of the commands from the various behaviors.

In [15], we use a finite state system to handle the outputs from different behaviors. For a behavior-based control system, however, the subsumption architecture has more advantages [21]. First, the finite state system, describing logic relationships between the behaviors, works as an arbitrator like an inhibition mechanism in the architecture, whereas the subsumption architecture describes a functionality of the behavior-based control system, e.g., how perception and action modules in the architecture are grouped to form individual behaviors with competence. Second, the subsumption architecture provides a bottom-up method of implementing the behavior-based control system layer-by-layer, so the subsumption architecture has easily expanded from having four behaviors in [14] to having six behaviors proposed herein. Next, the finite state system cannot be expected to handle outputs from different behaviors effectively, whose conflicts are necessarily coordinated by a suppression or a fusion mechanism [20].

\section{Design of Declare-Source Behavior}

\section{A. Multiple sensors for source declaration}

Factors that complicate source declaration include the chemical source concentration being unknown, the advection distance of any detected chemical being unknown, and the flow variation with both location and time. Therefore, multiple

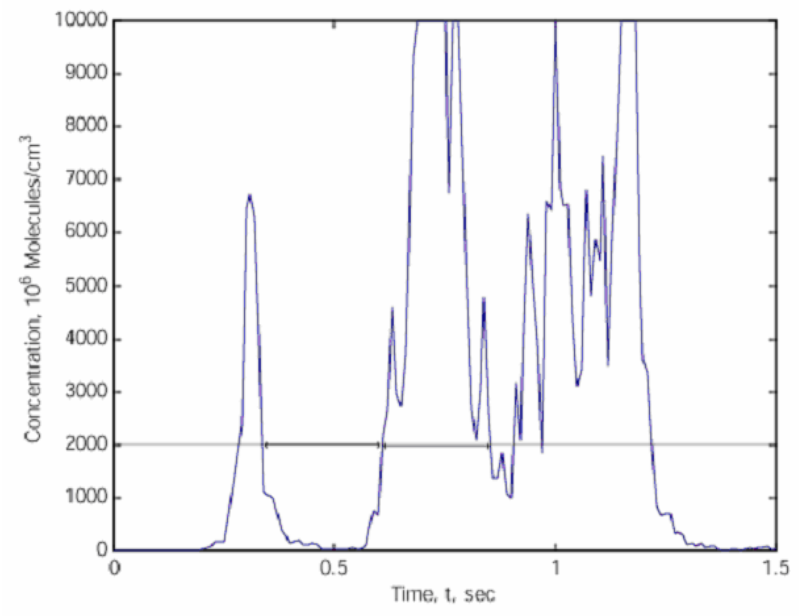

Fig. 2. Chemical concentration time series with threshold. 
sensors are required for the source declaration.

The first type of sensor is a fluorometer used to detect above threshold chemical concentration. Fig. 2 shows a typical example of the sensed chemical concentration resulting from a simulated model [22]. At medium and high numbers, the evolution of the chemical distribution in the flow is turbulence dominated [22]-[24]. The turbulent nature of transport mechanism causes the sensed concentration to be only intermittently above a positive sensor threshold. Such intermittency has several consequences. First, algorithms based on instantaneous gradient-following in an intermittent plume would be inefficient. The instantaneous gradient varies too rapidly to be calculated. Second, approaches to inferring the proximity of the source based on changes in concentration or to declaring the source based on a maximum concentration must be very carefully designed so as to work reliably, because a local maximum or a relatively high proximity of chemical

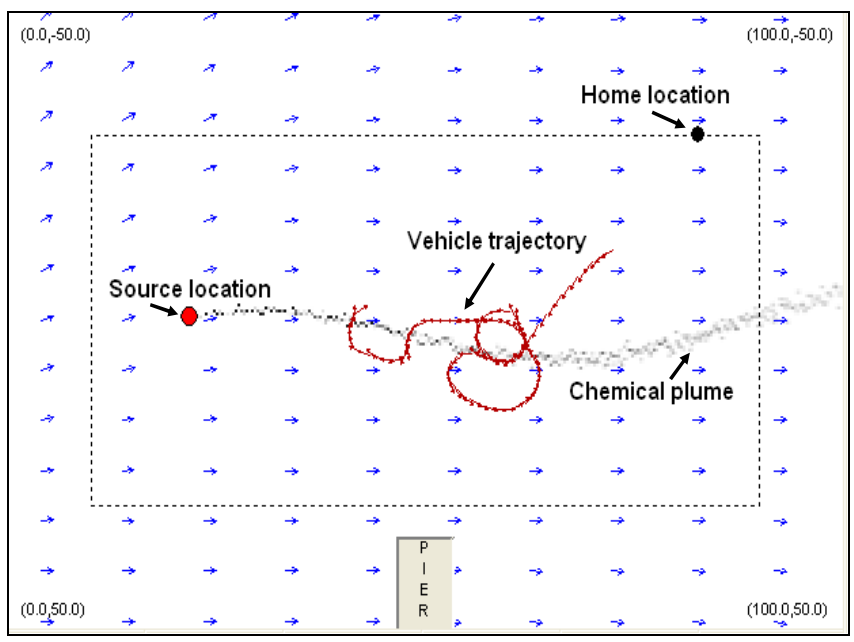

(a). AUV starts a CPT mission from the home location, including plume finding and plume tracing.

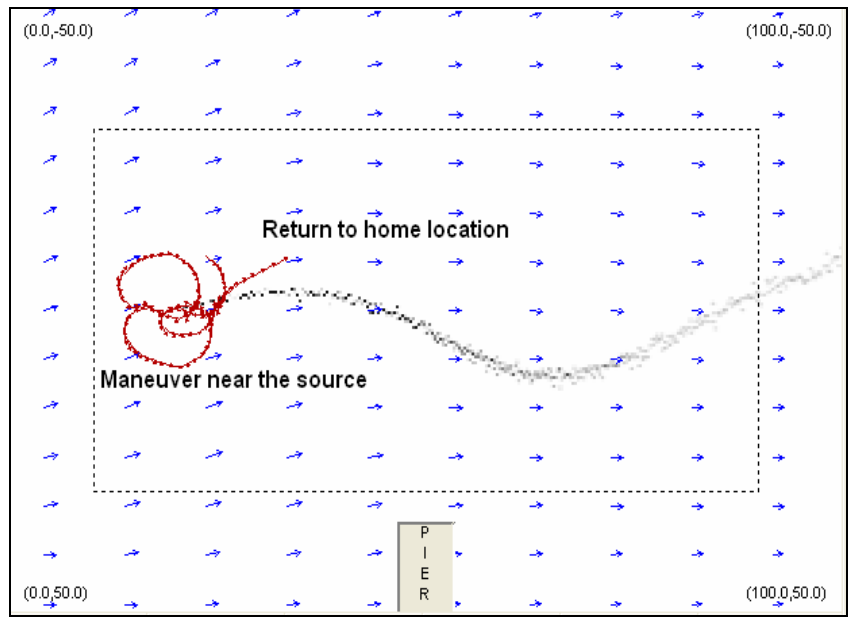

(b) AUV returns home after declared the source location.

Fig. 3. The head-to-tail arrows indicate the vehicle trajectory. The grayscale indicates above threshold concentration. The arrows in the operation area indicate the magnitude and direction of the local flow vector at the tail of the arrow.

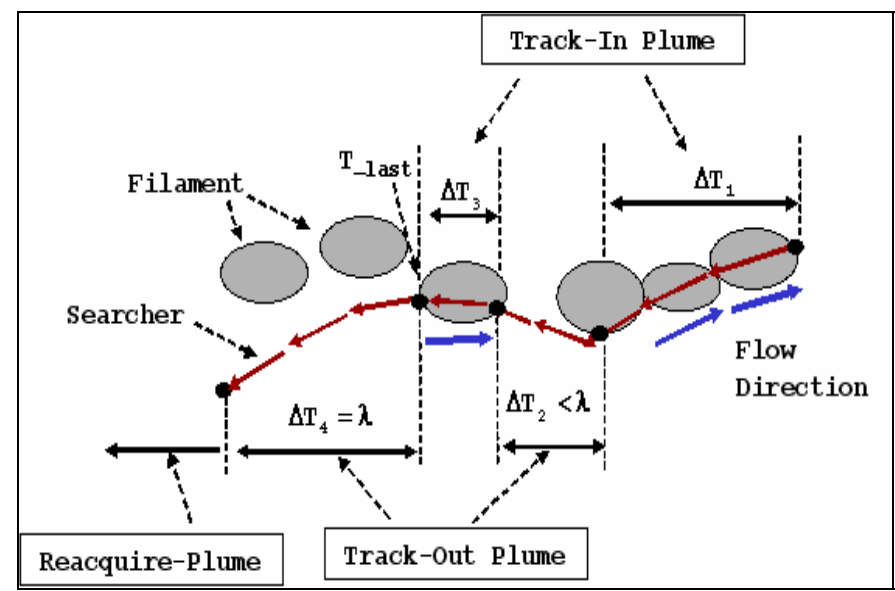

Fig. 4. Track-In and Track-Out activities in the Maintain-Plume behavior.

detection points above threshold can occur in all part of a plume developed in the turbulent fluid-advected environment. A sample rate of the AUV control system also affects the maximum or the proximity. Third, intermittency causes the concepts of "in the plume" and "not in the plume" to be difficult to define, when a plume tracing strategy is designed. In the proposed strategy, the chemical sensor works as a "binary detector". The Boolean value is "1" if the chemical concentration is above the threshold, while the Boolean value is " 0 if the chemical concentration is below the threshold.

The second kind of sensor for source declaration is used to provide information about instantaneous fluid flow velocity and direction, which vary spatially and temperately, as shown in Fig. 3. A chemical plume is developed in the fluid flow field in three modes: fluid advection, slow growth of the filaments, and centerline relative diffusion [22]. Fig. 3 displays a plume that results when advection, centerline relative diffusion, and filament growth are all active. In the proposed strategy, instantaneous flow direction is used to trace a chemical plume.

The next kind of sensor is used to measure AUV positions during CPT missions. Information about the AUV locations is required to estimate the odor source in combination with chemical concentrations and to report the coordinates of the declared odor source. In addition, a visual sensor is recommended to recognize the source odor, when the source location is declared.

\section{B. SIZ T based source declaration algorithm}

Declare-Source is to estimate and declare the chemical source location. The declaration must be accurate and reliable in the sense that it makes no false declarations. Being different from Maintain-Plume and Reacquire-Plume, there is no clear analog to the AUV declare source behavior for male moths. The idea of designing the source declaration algorithm is to dynamically construct a SIZ_T by LCDPs, which are maintained in a list. This algorithm relies on the plume tracing strategy, consisting of two moth-inspired behaviors: MaintainPlume and Reacquire-Plume [2]. Maintain-Plume is broken 
down into Track-In and Track-Out activities due to the intermittency of a plume transported in a turbulent fluid flow environment. The AUV alternatively utilizes Maintain-Plume and Reacquire-Plume in making progress towards the source location in the up-flow direction. That means, once the vehicle detects a plume, it activates Track-In ( $\Delta \mathrm{T}_{1}$ and $\Delta \mathrm{T}_{3}$ in Fig. 4) and continues Tack-Out ( $\Delta T_{2}$ in Fig. 4) when the AUV loses a contact with the plume within $\lambda$ seconds. After $\lambda$ seconds $\left(\Delta \mathrm{T}_{4}\right.$ in Fig. 4), it switches to Reacquire-Plume for casting the plume again based on Cloverleaf trajectories, as shown in Fig. 5. A chemical detection point, where the AUV loses the contact with the chemical for $\lambda$ seconds, is defined as a last chemical detection point (LCDP), $\left(x_{\text {last }}, y_{\text {last }}\right)$, at time $\mathrm{T}_{-}$last in Fig. 4. The important fact is: LCDPs are separated along the axis of the plume, when the AUV is far from the source location, while LCDPs get closer and are located near but down-flow from the odor source, when the AUV is approaching near the source. Fig. 5 shows a pattern for LCDPs distribution during CPT missions.

When the AUV switches its behaviors from Maintain-Plume to Reacquire-Plume, a new LCDP is created and inserted into the list, and a certain amount of the most recent LCDPs are maintained in the order of the time when they are detected. The size of SIZ_T is built by

$$
\begin{aligned}
& x_{\text {last }}^{(\min )}=\min \left\{x_{\text {last }}^{t(i)}\right\} \\
& x_{\text {last }}^{(\text {max })}=\max \left\{x_{\text {last }}^{t(i)}\right\} \\
& y_{\text {last }}^{(\min )}=\min \left\{y_{\text {last }}^{t(i)}\right\} \\
& x_{\text {last }}^{(\text {max })}=\max \left\{y_{\text {last }}^{t(i)}\right\}
\end{aligned}
$$

where a superscript $t$ is used to indicate that LCDPs are sorted in the order of time series. The mean value of the LCDPs

$$
\begin{aligned}
& \bar{x}_{\text {last }}^{t}=\sum_{i=1}^{N_{d e c}} x_{\text {last }}^{t(i)} / N_{\text {dec }} \\
& \bar{y}_{\text {last }}^{t}=\sum_{i=1}^{N_{d e c}} y_{\text {last }}^{t(i)} / N_{\text {dec }}
\end{aligned}
$$

is declared as the odor source, if the diameter of SIZ_T

$$
\sqrt{\left(x_{\text {last }}^{(\max )}-x_{\text {last }}^{(\min )}\right)^{2}+\left(y_{\text {last }}^{(\max )}-y_{\text {last }}^{(\min )}\right)^{2}} \leq \varepsilon_{T} .
$$

\section{Monte CARlo Evaluation}

Monte Carlo simulation studies are used to verify the effectiveness of the proposed strategy for declaring source locations in a simulated turbulent fluid environment, as shown in Fig. 3. The operation area is specified by $[0,100] \times[-50,50]$

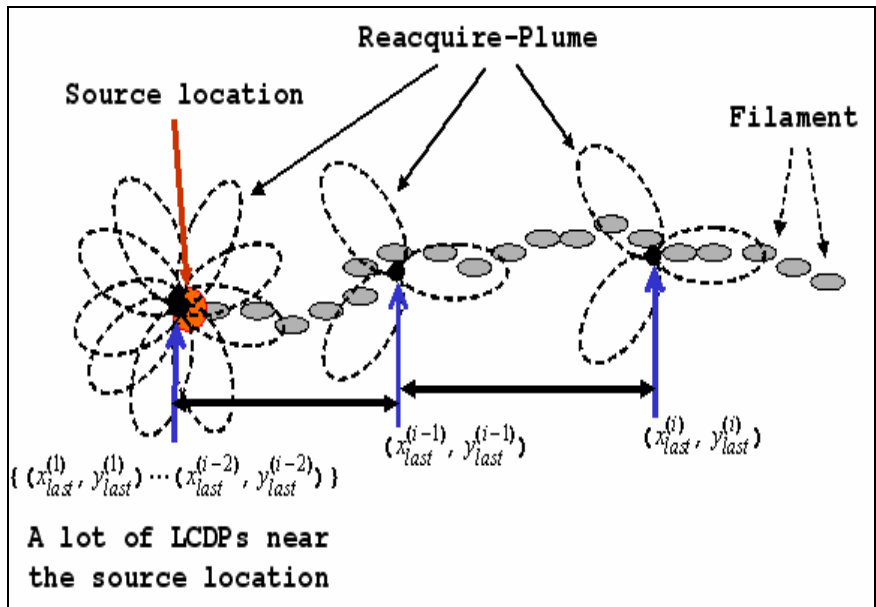

Fig. 5. Pattern for the source identification based on LCDPs in the order of the up-flow direction, which is assumed from left to right.

in meter. The filament release rate is 10 filaments $\mathrm{s}^{-1}$, the simulation time step is $0.01 \mathrm{~s}$, and the mean fluid velocity $2 \mathrm{~m}$ $\mathrm{s}^{-1}$. During the simulation studies, the measured fluid flow is corrupted by additive noise that is white normal random process. The vehicle home location is defined as $(80,-30) \mathrm{m}$ in the operation area. The coordinates of a "true" source location are chosen as $(20,0) \mathrm{m}$, which are just used to check the accuracy of the declared source locations, but unknown to the AUV during CTP missions. The simulation time is limited within $T_{\max }=1000$ seconds.

A CPT mission is defined as follows: The vehicle starts from the home location and utilizes Find-Plume, MaintainPlume and Reacquire-Plume activities to find a plume, trace the plume towards the source location. The time cost for a source declaration activity is defined by the period from the time when the AUV reaches a point within $10 \mathrm{~m}$ to the "true" source location to the time when the odor source is declared. The AUV returns home after it declares the odor source, and then this CPT mission completes, as shown in Fig. 3. A CPT mission fails, if the AUV could not declare the source location within $T_{\max }$. The simulation studies are to continuously perform 1000 CPT missions, which keep changing a plume in a fluid-advected environment over 100 hours. The parameters of the strategy are selected as $\varepsilon_{T}=6$ meters and $N_{\mathrm{dec}}=6$. Table I documents the results of these missions in the three aspects: reliability, accuracy, and time cost. A success rate in declaring the source location is about $98.4 \%$. In the 1000 test runs, the strategy has 6 spurious declarations and 14 "over time limit" runs. A spurious declaration is defined by a declared source

TABLE I

Performance of Source DeClaration Alogorithm

\begin{tabular}{|l|l|l|l|l|l|l|l|}
\hline \hline Parameters & Success rate & $\begin{array}{l}\text { Spurious declaration } \\
\text { / over limit }\end{array}$ & $\begin{array}{l}\text { Source error, } \\
\text { meters }\end{array}$ & $\begin{array}{l}\text { Error within } \\
\text { 2 meters }\end{array}$ & $\begin{array}{l}\text { Error within } \\
2-5 \text { meters }\end{array}$ & $\begin{array}{l}\text { Error within } \\
5-10 \text { meters }\end{array}$ & $\begin{array}{l}\text { Time cost for declaration } \\
\text { and mission, seconds }\end{array}$ \\
\hline \hline$\varepsilon_{T}=6 \mathrm{~m}$ & $\begin{array}{l}980 \text { of } 1000 \\
(98.0 \%)\end{array}$ & $\begin{array}{l}6 \text { of } 1000 / \\
10 \text { of } 1000\end{array}$ & $\begin{array}{l}\Delta \overline{\mathbf{x}}=3.27 \mathrm{~m} \\
\Delta \overline{\mathbf{Y}}=0.00 \mathrm{~m}\end{array}$ & $\begin{array}{l}86 \text { of } 980 \\
(8.78 \%)\end{array}$ & $\begin{array}{l}802 \text { of } 980 \\
(81.84 \%)\end{array}$ & $\begin{array}{l}91 \text { of } 980 \\
(9.29 \%)\end{array}$ & $\begin{array}{l}\overline{\mathrm{T}}_{\mathrm{D}}=218.48 \mathrm{~s} \\
\overline{\mathrm{T}}_{\mathrm{T}}=387.98 \mathrm{~s}\end{array}$ \\
\hline
\end{tabular}




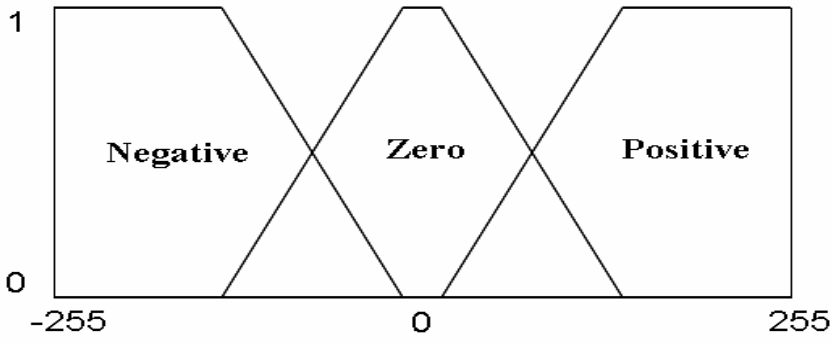

(a). Fuzzy sets for color difference

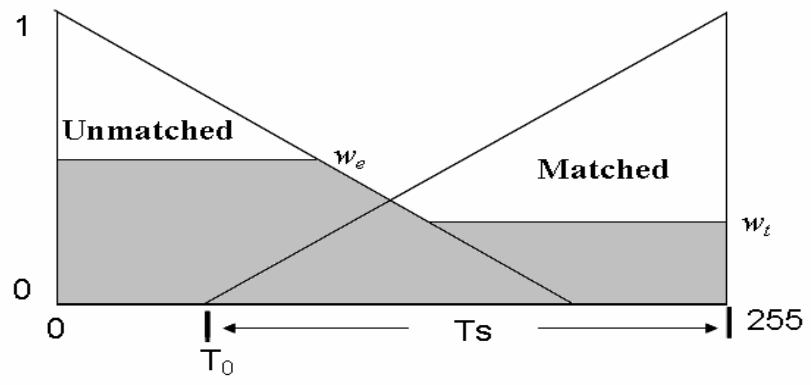

(b) Fuzzy sets for defuzzification

Fig. 6. fuzzy-reasoning based color segmentation algorithm.

location with a distance to the "true" source location is greater than $10 \mathrm{~m}$. The average accuracy of the declared source locations is about $3.27 \mathrm{~m}$. Columns 5-7 in Table I also show a distribution of the declared source locations in three groups: within $2 \mathrm{~m}$, between 2 and 5 meters, and between 5 and $10 \mathrm{~m}$. The strategy reaches a rate of the declared source locations $88.2 \%$ within $2-5$ meters, for the 1000 CPT missions. The average time cost for the odor source declarations is $218.48 \mathrm{~s}$, and the total time cost is $387.98 \mathrm{~s}$.

\section{SOURCE VerificATION}

One version of source declaration algorithms was implemented on an AUV for in-water tests [14], [15]. Fig. 7a shows a scenario about the chemical plume and its source taken by a camera system while the vehicle was approaching to the odor source. It is proposed to design a Source-Verification module to allow visual confirmation the correct declared odor source interactively or automatically. When the AUV approaches the declared odor source, this module takes a picture and tries to verify the odor source. Because the vehicle operates in near-shore ocean environments with uncertainty, fuzzy reasoning based image segmentation is recommended in [25]. The following brief discussion addresses a fuzzyreasoning based color segmentation algorithm for SourceVerification [26]. The first step of the algorithm is extraction of the components of red, green, and blue in an image. Then, for each component value, the differences between a component value and a pattern value are computed. These differences are inputs to the fuzzy sets, as shown in Fig. 6a. After fuzzy reasoning, the weights for the sets Matched and Unmatched are determined in Fig. 6b. Finally, the plume or

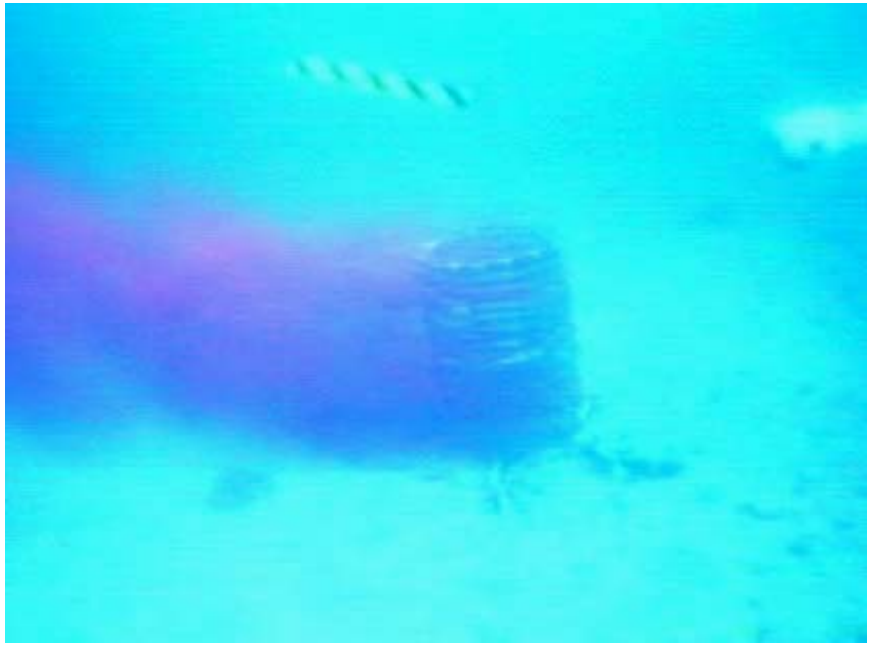

(a). Scenario about the plume and its source was taken by a camera system while the AUV was approaching to the source.

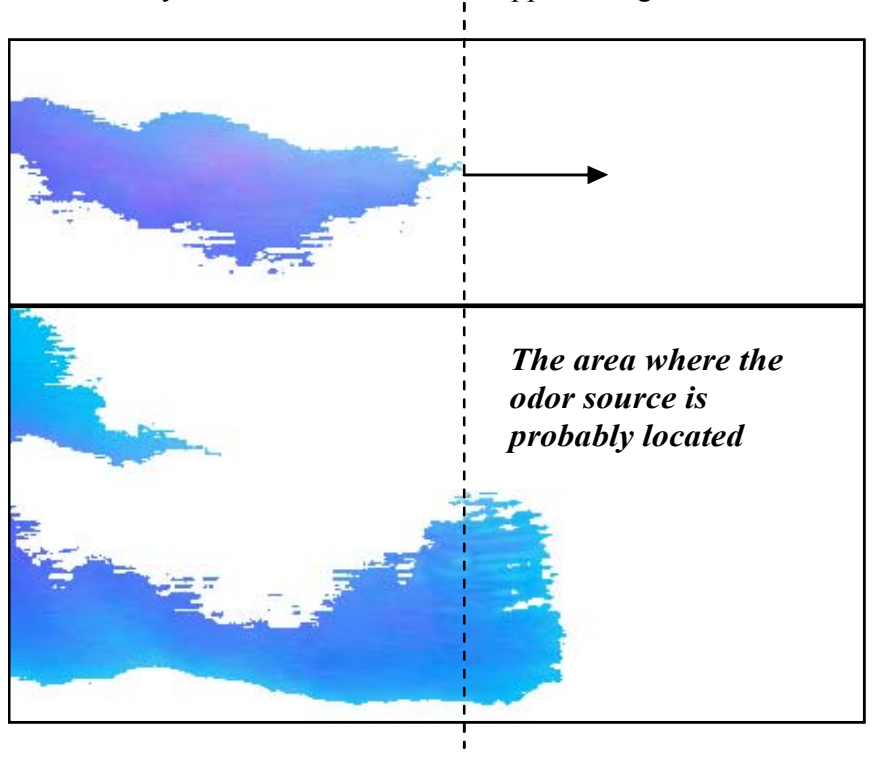

(b). Extracted color components of the chemical plume (top) and extracted color components of the odor source (bottom).

Fig. 7. Plume source recognition by Source-Verification

the odor source is segmented from the image. Fig $7 \mathrm{~b}$ illustrates the extracted color components of both the plume and the odor source, respectively. Such information would be useful in recognizing the plume source, because the fluid-advected chemical plume ends its source location. More detail discussions about this algorithm will be addressed in our further work.

\section{DISCUSSIONS AND CONCLUSION}

CPT in the real world is a very challenging task. Obstacle avoidance should be considered during CPT. Usually, in traditional path planning, a target for a robot can be specified in advance, but in CPT mission, there is no any information about a chemical plume and its odor source available before CPT missions. Especially, in an operation area with large scales, strategies for efficiently finding a plume and tracing the 
plume in presence of obstacles must be developed. The field tests show that, avoiding soft obstacles, such kelp forests or seaweed in deep water, is more serious than avoiding hard obstacles, because some filaments of the chemical can be transported among gaps in kelp forests, which cause the AUV to become stuck during CPT missions in [20].

Sensing soft obstacles makes another significant challenge to the current marine sensors. Sonar sensors, which are able to detect hard obstacles, do not work for soft obstacles well. For CPT in near-shore ocean conditions, an AUV operates in deep water from few meters to over twenty meters. A visual sensor is impractical to detect soft obstacles in this case, because the AUV must illuminate dark environments by a very powerful lighting system during CPT missions. However, a small and light underwater vehicle has very limited power. A visual sensor is just recommended to verify the declared odor source when the source location is declared.

Chemical plume tracing in near shore ocean conditions is a three dimensional (3-D) problem. In particular, finding a plume in $3 \mathrm{D}$ is very time consuming, because there is no any information about the plume prior to CPT missions. However, neutral buoyancy of the chemical [27] or stratification of the flow [28] will often result in a plume of limited vertical extent, which may be approximated as a two dimensional (2-D) problem. In the field tests, the AUV was so controlled that operated in a fixed altitude terrain-following mode intended to keep the vehicle in the bottom boundary layer, and no test runs failed due to the 3D problem.

Reverting back to Find-Plume can be avoided when the AUV fails to re-contact the chemical plume by ReacquirePlume, if Construct-Plum-Map is integrated. However, the current test runs are intent to evaluate performance of FindPlume, because Find-Plume is hard to study.

\section{ACKNOWLEDGMENT}

Jay A. Farrell, Ring Cardé and John Murlis were key collaborators while developing the Chemical Plume Tracing strategy.

\section{REFERENCES}

[1] J. H. Belanger and M. A. Willis, "Adaptive control of chemical -guided location: Behavioral flexibility as an antidote to environmental unpredictability," Adaptive Behavior, vol. 4, pp. 217-253, 1998.

[2] W. Li, J. A. Farrell, and R. T. Cardé, "Tracking of fluid-advected chemical plumes: Strategies inspired by insect orientation to pheromone," Adaptive Behavior, vol. 9, pp. 143-170, 2001.

[3] F. W. Grasso, T. R. Consi, D. C. Mountain, and J. Atema, "Biomimetic robot lobster performs chemo-orientation in turbulence using a pair of spatially separated sensors: Progress and challenges," Robotics and Autonomous Systems, vol.30, pp. 115-131, 2000.

[4] F. W. Grasso and J. Atema, "Integration of flow and chemical sensing for guidance of autonomous marine robots in turbulent flows," Journal of Environmental Fluid Mechanics, vol. 1, pp. 1-20, 2002.

[5] J. A. Farrell, S. Pang, and W. Li, "Plume mapping via Hidden Markov methods," IEEE Trans on Systems, Man, and Cybernetics-Part B: Cybernetics, vol. 33, pp. 850-863, 2003.

[6] R. A. Russell, D. Thiel, R. Deveza, and A. Mackay-Sim, "A robotic system to locate hazardous chemical leaks," in Proc. of 1995 IEEE Inter. Conf. on Robotics and Automation," vol. 1, pp. 556-561, 1995.
[7] H. Ishida, T. Nakamoto, T. Moriizumi, T. Kikas, and J. Janata, "Plumetracking robots: A new application of chemical sensors," Biological Bulletin, vol.200, pp. 222-226, 2001.

[8] Y. Kuwana, S. Nagasawa, I. Shimoyama, and R. Kanzaki, "Synthesis of the pheromone-oriented behavior of silkworm moths by a mobile robot with moth antennae as pheromone sensors," Biosensors \& Bioelectronics, vol.14, pp. 195-202, 1999.

[9] S. Kazadi, R. Goodman, D. Tsikata, and H. Lin, "An autonomous water vapor plume tracking robot using passive pesistive polymer sensors," Autonomous Robots, 9(2), pp. 175-188, 2000.

[10] L. Marques, U. Nunes, and A. T. de Almeida, "Olfaction-based mobile robot navigation," Thin Solid Films, vol. 418, p. 51-58, 2002.

[11] A. T. Hayes, et al., "Distributed chemical source localization," IEEE Sensors Journal, vol. 2, pp. 260-271, 2002.

[12] D. Zarzhitsky, D. F. Spears, and W. F. Spears, "Distributed robotics approach to chemical plume tracing," in Proc. of Int. Conf. on Intelligent Robots and Systems, 2005

[13] V. N. Christopoulos and S. Roumeliotis, "Multi robot trajectory generation for single source explosion parameter estimation", in Proc. of IEEE Conf. on Robotics and Automation, pp.2184-2820, 2005.

[14] W. Li, J. A. Farrell, S. Pang, and R. M Arrieta, "Moth-inspired chemical plume tracing on an autonomous underwater vehicle", IEEE Transactions on Robotics, vol.22, no.2, pp.292-307, 2006.

[15] J. A. Farrell, S. Pang, and W. Li, "Chemical plume tracing via an autonomous underwater vehicle," IEEE Journal of Ocean Engineering. vol. 30, pp.428-442, 2005.

[16] R. T. Cardé and A. Mafra-Neto, "Mechanisms of flight of male moths to pheromone." In R.T. Cardé and A.K. Minks (eds.), Insect Pheromone Research. New Directions, Chapman and Hall, New York, pp. 275-290, 1996.

[17] L. P. S. Kuenen and R. T. Cardé, "Strategies for recontacting a lost pheromone plume: Casting and upwind flight in the male gypsy moth," Physiological Entomology, vol.19, pp. 15-29, 1994.

[18] M. W. Sabelis and P. Schippers, "Variable wind directions and anemotactic strategies of searching for an odour plume," Oecologia, vol. 63, pp.225-228, 1984.

[19] D. B. Dusenbery, "Optimal search direction for an animal flying or swimming in a wind or current," Journal of Chemical Ecology, vol.15, pp.2511-2519, 1989.

[20] W. Li and D. Carter, "Subsumption architecture for fluid-advected chemical plume tracing with soft obstacle avoidance" in Proc. of Ocean 2006 Marine Technology and Ocean Science Conf., (in press) 2006.

[21] R. A. Brooks, "A robust layered control system for a mobile robot," IEEE J. Robot. Automation, vol. 2, pp. 14-23, 1986.

[22] J. A. Farrell, J. Murlis, W. Li, and R. T. Cardé, "Filament-Based Atmospheric Dispersion Model to Achieve Short Time-Scale Structure of Chemical Plumes", Journal of Environmental Fluid Mechanics, vol. 2, pp. 143-169, 2002.

[23] D. R. Webster and M. J. Weissburg, "Chemosensory guidance cues in a turbulent chemical odor plume," Limnology Oceanography, 46(5), pp.1034-1047, 2001.

[24] J. Murlis, "The structure of odour plumes," In T. L. Payne, M. C. Birch, and C. E. J. Kennedy (eds.), Mechanisms in insect olfaction, pp.27-38, 1986.

[25] W. Li, F. M. Wahl, J. Z. Zhou, H. Wang, and K. Z. He, "Vision-based behavior control of autonomous systems by fuzzy reasoning," Modeling and Planning for Sensor-Based Intelligent Robot Systems, pp.311-325, Springer-Verlag, Germany, 1999.

[26] M. M. Elgassier, "Color recognition with fuzzy segmentation," Senior Seminar Project, Department of Computer Science, California State University, Bakersfield, Fall, 2005.

[27] H. Schlicting and K. Gersten, Boundary-layer theory, $8^{\text {th }}$ Edition, Springer-Verlag, New York, pp. 29-48, 2000.

[28] M. T. Stacey, E. A. Cowen, T. M. Powell, E. Dobbins, S. G. Monismith, and J. R. Koseff, "Plume dispersion in a stratified, near-coastal flow: measurements and modeling", Continental Shelf Research, vol. 20, pp.637-663, 2000. 